University of Wollongong

Research Online

Faculty of Engineering and Information

Faculty of Engineering and Information

Sciences - Papers: Part A

Sciences

$1-1-2014$

\title{
A multiple wavelength unwrapping algorithm for digital fringe profilometry based on spatial shift estimation
}

Pu Cao

University of Wollongong, pc241@uowmail.edu.au

Jiangtao Xi

University of Wollongong, jiangtao@uow.edu.au

Yanguang Yu

University of Wollongong, yanguang@uow.edu.au

Qinghua Guo

University of Wollongong, qguo@uow.edu.au

Follow this and additional works at: https://ro.uow.edu.au/eispapers

Part of the Engineering Commons, and the Science and Technology Studies Commons

Research Online is the open access institutional repository for the University of Wollongong. For further information contact the UOW Library: research-pubs@uow.edu.au 


\title{
A multiple wavelength unwrapping algorithm for digital fringe profilometry based on spatial shift estimation
}

\author{
Abstract \\ In this paper, a new approach is presented for solving the problem of spatial shift wrapping associated \\ with Spatial Shift Estimation (SSE)-based Fringe Pattern Profilometry (FPP). The problem arises as the \\ result of fringe reuse (that is, fringes periodic light intensity variance), and the spatial shift can only be \\ identified without ambiguity with the range of a fringe width. It is demonstrated that the problem is similar \\ to the phase unwrapping problem associated with the phase detection based FPP, and the proposed \\ method is inspired by the existing ideas of using multiple images with different wavelengths proposed for \\ phase unwrapping. The effectiveness of the proposed method is verified by experimental results on an \\ object with complex surface shape. 2014 SPIE.

\section{Keywords} \\ fringe, digital, algorithm, unwrapping, profilometry, wavelength, estimation, multiple, spatial, shift

\section{Disciplines} \\ Engineering | Science and Technology Studies

\section{Publication Details} \\ P. Cao, J. Xi, Y. Yu \& Q. Guo, "A multiple wavelength unwrapping algorithm for digital fringe profilometry \\ based on spatial shift estimation," in Proceedings of SPIE 9013: Three-Dimensional Image Processing, \\ Measurement (3DIPM), and Applications 2014, 2014, pp. 90130E-1-90130E-10.
}




\title{
A Multiple Wavelength Unwrapping Algorithm for Digital Fringe Profilometry based on Spatial Shift Estimation
}

\author{
$\mathrm{Pu}$ Cao, Jiangtao Xi*, Yanguang Yu and Qinghua Guo \\ School of Electrical, Computer and Telecommunications Engineering \\ University of Wollongong, Wollongong, NSW2522, Australia
}

\begin{abstract}
In this paper we present a review of the phase unwrapping problem in Fringe Pattern Profilometry (FPP), based on which we study the spatial shift wrapping problem in spatial shift estimation (SEE) based FPP. An approach for carrying out the spatial shift unwrapping is proposed with its performance confirmed by experiments.
\end{abstract}

Keywords: fringe pattern profilometry, 3D measurement, phase unwrapping

\section{Introduction:}

Fringe Pattern Profilometry (FPP) based on Digital Fringe Projection (DFP) is a promising optical noncontact threedimension (3D) profile measurement technologies due to its accuracy and flexibility. Compared with the other methods, it has the advantages of simple system structure and high accuracy. Hence it provides a much more flexible and practical approach for 3D profile measurement.

Figure 1 shows the system structure of a DFP based FPP, consisting of a digital video projector, a CCD camera and a reference plane. With the system, a frame of image with a particular fringe pattern is produced by the digital projector and projected onto the reference plane, and then onto the surface of the object when the reference plane is removed. The projected images from the reference plane and the object surface are captured by the CCD camera, with the later being a deformed version of the former by the variance of the height of the object surface. As the deformed fringe pattern carries the information of surface shape, 3D profile of the object can be retrieved from these two fringe patterns.

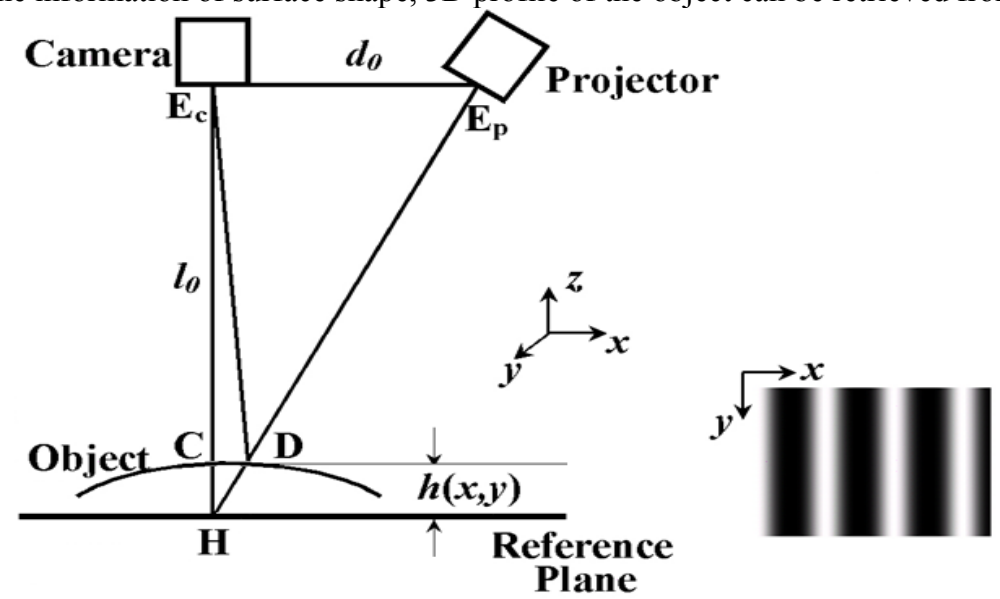

Figure 1. Schematic diagram of FPP system. 
Several FPP approaches have been developed during the past decades. The most widely used are these based on phase difference estimation (PDE). In these approaches, the projected fringe patterns are sinusoidal or periodic, and the deformed one reflected from the object surface is considered as the result of phase modulation of the original fringe pattern. The surface profile is obtained by detecting the phase maps of the two fringe patterns. A number of fringe pattern analysis methods have been developed, such as Fourier transform profilometry (FTP) [1], phase shifting profilometry (PSP), phase measuring profilometry (PMP) [2, 3, 4], modulation measurement profilometry (MMP) [5], spatial phase detection (SPD) [6,7], phase lock loop (PLL) profilometry [8], Moire technique (MT) [9], laser triangulation measurement [10], colour-coded fringe projection [11, 12] and other methods [13, 14]. Among existing approaches, FTP and PSP are most popular and widely used.

Although phase based approaches have been considered as the most popular, they suffers from a number of weaknesses. A major restriction is that fringe patterns must be either sinusoidal or ideal periodic. However such a requirement is hard to meet in practice due to some factors, such as the nonlinear distortion on inherent to digital video projections. In order to solve the problem, a profilometry approach was proposed by $\mathrm{Hu}$ et al $[15,16,17]$, which, instead of detecting the differences between the phase maps, is based on the estimation of spatial shift for corresponding pixels on the two fringe patterns. The approach is referred to as spatial shift estimation (SSE) profilometry approach.

The spatial shift estimation approach is suitable for any fringe pattern, however, use of a periodic fringe patterns is still necessary in order to have enough measurement resolution. In the spatial shift estimation-based approaches, spatial shift between corresponding pixels on the two fringe patterns is arbitrary, it can only be detected without ambiguity within the range of $[0, \lambda]$, where $\lambda$ is the wavelength, or the spatial width of the individual fringe, i.e., number of pixels per fringe stripe. Obviously, shift unwrapping is also required in order to correctly restore the 3D shape of the object surface. However, spatial shift unwrapping for complex object using spatial shift estimation-based fringe pattern profilometry is still an outstanding issue, which motivated the work presented in this paper.

Since the spatial shift unwrapping problem exists in spatial shift estimation approach which is similar to the phase unwrapping problem in phase difference estimation based fringe pattern profilometry, a review of the phase unwrapping problem is given in this paper. Phase unwrapping problem is a major problem associated with phase difference estimation-based fringe pattern profilometry approaches. This problem arises because the phase difference can only be detected within the main value range of $[-\pi, \pi]$, but the true phase difference can be arbitrary. In order to retrieve the actual surface shape of the object, phase unwrapping must be carried out to obtain the actual phase maps. To solve the unwrapping problem in phase difference estimation approach, Zhang et al [18] introduced a multiple wavelength phase unwrapping algorithm. In his method, an image which only has a single fringe covers the whole measurement area is first projected, and then a series of fringe images with a wavelength decreased by a factor from its previous wavelength is used. Since the first image only contains one fringe, the unwrapping step is not required. Then the phase of second image can be unwrapped by referring the first image. After the phase of second image is obtained, it can be used to correct the third image. In general, the phase of each wavelength is unwrapped by referring to the unwrapped longer wavelength phase pixel by pixel.

Based on Zhang's method, we introduce a multiple wavelength unwrapping algorithm for spatial shift estimation approach. In our method, a series of fringe images with a wavelength decreased by a factor from its previous wavelength is also applied. The spatial shift of each wavelength is then unwrapped by referring to the unwrapped longer wavelength spatial shift pixel by pixel. Since the longest wavelength covers the whole measurement area, no spatial shift unwrapping step is necessary. The proposed method solves the spatial shift unwrapping problem in spatial shift estimation, hence it can enable the measurement of complex objects with significant step height or multiple separate objects using spatial shift estimation approach.

This paper is organized as follows. In Section 2 we firstly give a brief introduction on the conventional phase difference estimation based fringe pattern profilometry and the spatial shift estimation based technique, including their principles, system structures and relevant algorithms. Then in Section 3 we indicate that the unwrapping problem exists in spatial shift estimation approach which is similar to the phase unwrapping problem in phase difference estimation based fringe pattern profilometry. The paper then gives a review of the multiple wavelength phase unwrapping algorithm introduced by Zhang, based on which we introduce a multiple wavelength unwrapping algorithm for spatial shift estimation approach. Finally in Section 4 experimental results are given to demonstrate the proposed method can be used to measure complex objects with significant step height or multiple separate objects using spatial shift estimation approach.

\section{Principle of Fringe Pattern Profilometry}




\subsection{Principle of Triangulation}

FPP is based on the triangulation principle described as follows. As the image produced by the projector has a fringe structure, without loss of generality we can assume that light intensity varies periodically alone $x$ direction, while keeping constant along $y$ direction, as shown in Figure 1. We can use $s(x), d(x)$ and $h(x)$ to denote the variance of light intensity of the fringe pattern on the reference plane and object surface as well as the height distribution along $x$ coordinate respectively. We also assume that the reference plane and the object surface have the same reflective characteristics.

Let us consider what happens when a beam of light is projected onto the point $\mathrm{D}$ on the object. When the object is removed, the same light beam (hence with the same intensity) should be projected onto point $\mathrm{H}$ on the reference surface, which is reflected back to the camera through point $\mathrm{C}$. As the triangles $E_{c} E_{P} H$ and $C D H$ are similar, we have the following relationship:

$$
\frac{d_{0}}{l_{0}}=\frac{\overline{C D}}{h\left(x_{d}\right)}
$$

Note that $x_{d}$ denotes the coordination positions of point D. $h\left(x_{d}\right)$ denotes the distance between points $\mathrm{C}$ and the reference plane, given by:

$$
h\left(x_{d}\right)=\frac{l_{0} \overline{C D}}{d_{0}}
$$

The above relationship gives the foundation for FPP.

\subsection{PDE based approaches for FPP}

The PDE based FPP utilize fringe patterns that are periodic and can be expressed as [19, 20]:

$$
s(x)=\sum_{k=0}^{+\infty} b_{k} \cos \left(2 \pi k f_{0} x+\psi_{k}\right)
$$

and the deformed fringe pattern can also be expressed as:

$$
d(x)=\sum_{k=0}^{+\infty} b_{k} \cos \left(2 \pi k f_{0} x+\phi_{k}(x)+\psi_{k}\right)
$$

In the above equations, $f_{0}$ is the spatial frequency of the fundamental component in the fringe patterns, and $b_{k}$ is the amplitude of the $k$-th order harmonic component. $\psi_{k}$ is the initial phase of the $k$-th order harmonic component, and $\phi_{k}(x)$ denotes the phase difference between the $k$-th order harmonic components of these two fringe patterns.

Equations (3) and (4) show that $s(x)$ and $d(x)$ are related by the phase shift $\phi_{k}(x)$. Let us consider the light beam projected at point $\mathrm{D}$ on the object and $\mathrm{H}$ on the reference plane when the object is removed. The phase shift between $\mathrm{C}$ and $\mathrm{D}$ can be determined by the spatial distance $\overline{C D}$, and hence we have [21,22]:

$$
\phi_{k}\left(x_{d}\right)=2 \pi k f_{0} \overline{C D}=k \cdot 2 \pi f_{0} \overline{C D}=k \cdot \phi\left(x_{d}\right)
$$

where $\phi\left(x_{d}\right)=2 \pi f_{0} \overline{C D}$ is the phase shift of the fundamental component.

Substituting Equation (5) to Equation (2) we have:

$$
h\left(x_{d}\right)=\frac{l_{0} \phi\left(x_{d}\right)}{2 \pi f_{0} d_{0}}
$$

As points $\mathrm{D}$ and $\mathrm{H}$ are arbitrary, the derivations should apply to all the points on the projected fringe pattern. Therefore we have: 


$$
h(x)=\frac{l_{0} \phi(x)}{2 \pi f_{0} d_{0}}
$$

Equation (7) shows that as long as the gives $\phi(x)$ can be detected, we are able to calculate the height distribution $h(x)$ of the object surface. This is the foundation of all PDE based approaches.

\subsection{Spatial Shift Estimation based FPP}

The PDE based FPP methods suffer from some limitations. In particular, the fringe pattern used to project is limited to be sinusoidal or purely periodic in order that the phase maps of $s(x)$ and $d(x)$ exist and can be detected. However, due to many undesired factors inherent to digital projection, such as geometrical distortion and nonlinear intensity distortion, purely sinusoidal fringe patterns are hard to produce. In order to solve these problems, Hu et al [15] introduced a method which is based on the spatial shift estimation (SSE) rather than PDE.

The SSE based approach is rather simple and straight forward. Let us consider $\overline{C D}$, the distance between $\mathrm{C}$ and $\mathrm{D}$ again, which is obviously a function of the location of $\mathrm{D}$ (i.e. $x_{d}$,), the location of $\mathrm{H}$ (or C, i.e., $x_{c}$ ) and the height of the object at point $\mathrm{H} h\left(x_{d}\right)$. Therefore we have the following:

$$
\frac{d_{0}}{l_{0}}=\frac{u\left(x_{d}\right)}{h\left(x_{d}\right)}
$$

where $u\left(x_{d}\right)=\overline{C D}=x_{c}-x_{d}$, which is the spatial distance between $x_{d}$ and $x_{c}$. Note that $x_{d}$ and $x_{c}$ are the points on $d(x)$ and $s(x)$ having the same light intensity, that is $d\left(x_{d}\right)=s\left(x_{c}\right)$. As the above derivation is valid for any $x_{d}$ and $x_{c}$, we can replace $x_{d}$ by $x$, yielding the following:

$$
h(x)=\frac{l_{0} u(x)}{d_{0}}
$$

Note that $u(x)$ is the spatial distance between a point $x$ on $d(x)$ and the corresponding point on $s(x)$ with the same light intensity, that is:

$$
d(x)=s(x-u(x))
$$

Equations (9) and (10) provide a straight forward way to obtain the 3D profile of the object surface. With $d(x)$ and $s(x)$ available, if we are able to obtain $u(x)$ to meet Equation (10), we then can utilize Equation (9) to yield $h(x)$, the height distribution of the object surface along $x$. By repeating the procedure for all $y$ we should be able to obtain the $3 \mathrm{D}$ profile of the object surface.

A number of approaches were proposed to retrieve the $u(x)[1516,17]$. Among these approaches, the one referred to as Inverse Function based Shift Estimation (IFSE) [16] is particularly interesting and briefed as follows. Wu et al [23] then improved this method by combining IFSE with Jia's Multiple-step triangular-pattern phase shifting algorithm [24], which greatly improved the accuracy of measurement.

The spatial shift based approach has a particular advantage. The projected fringe patterns are no longer required to be sinusoidal, which implies that even there are distortions with the fringe patterns, sufficient three-dimensional information on the object surface is contained in the variation between projected and deformed fringe patterns. Thus the profilometry can be archived.

\section{The Unwrapping Problem}

As we know, with most PDE based approaches, $\phi(x)$ can only be identified within the range of $[-\pi, \pi]$. In other words, the phase is wrapped into the main value range. The real phase, $\Phi(x)$, should be continues as a function of $\phi(x)$ :

$$
\Phi(x)=2 \pi m(x)+\phi(x)
$$


where $m(x)$ are integers. This is called the wrapping problem, and the retrieving of $m(x)$ is called phase unwrapping.

\subsection{Spatial Shift Unwrapping in SSE based FPP:}

The wrapping problem also exists in SSE approaches. From Equation (9) we have:

$$
u(x)=\frac{d_{0} h(x)}{l_{0}}
$$

Depending on $h(x), d_{0}$ and $l_{0}$, the shift function $u(x)$ may take any value as well. However, when $s(x)$ has a fringe structure with a periodic fringe of width $\lambda, u(x)$ can only be detected within the main value of $[0, \lambda]$. In other words, $u(x)$ is wrapped into $[0, \lambda]$. Thus the real shift function, which is denoted as $U(x)$, should be continues as a function of $u(x)$ :

$$
U(x)=\lambda m(x)+u(x), \text { where } m(x) \text { are integers. }
$$

In order to demonstrate the relationship, we utilize the example in Figure 2. With $h(x)$ shown in Figure 2 (a), we should have $U(x)$ in Figure 2 (b). However, what we have is $u(x)$ as shown by Figure 2 (c). Use of $u(x)$ in Equation (9) will result in significant error in $h(x)$, as shown by Figure 2 (d). Therefore, we must work out a way to restore $U(x)$. The process is referred to as spatial shift unwrapping.

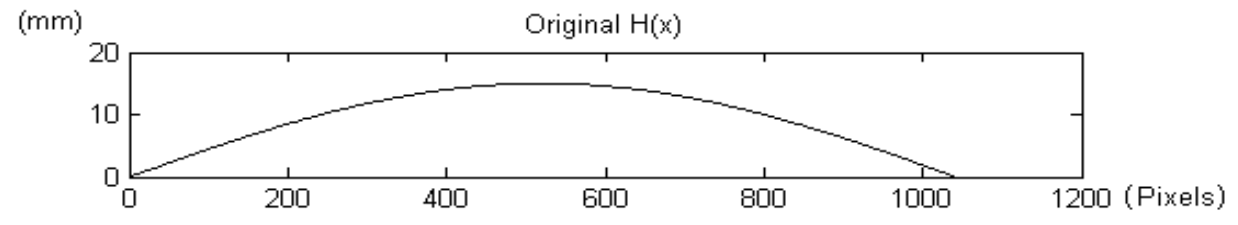

(a)
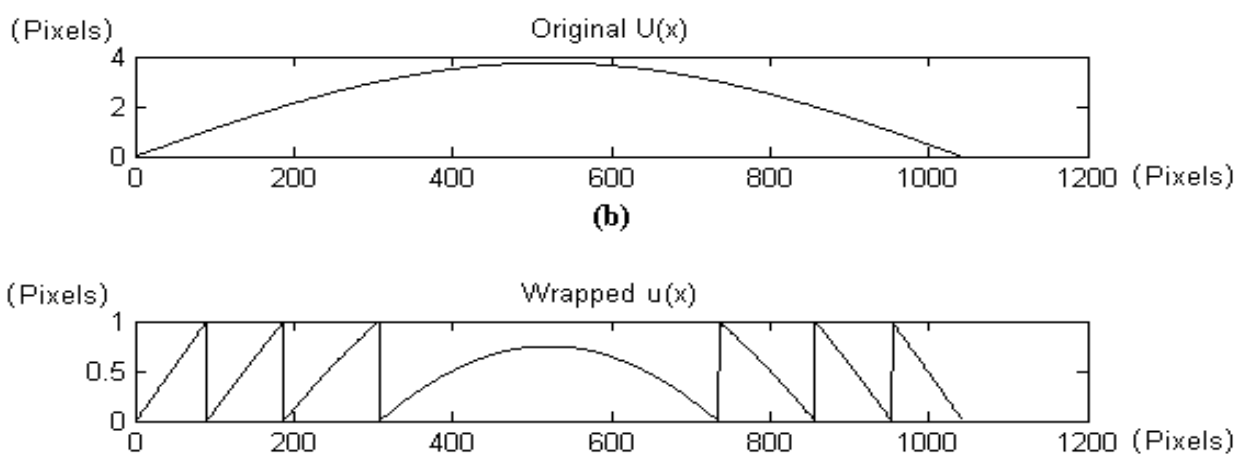

(c)

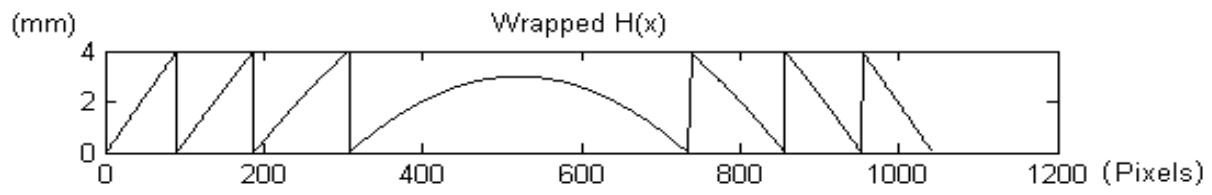

(d)

Figure 2. Unwrapped and wrapped shift maps 


\subsection{Multi-wavelength Phase Unwrapping Algorithm:}

To solve the phase unwrapping problem, Zhang et al [18] introduced a multiple wavelength phase unwrapping algorithm. In his method, a single fringe covers the whole measurement area is first projected. That is, suppose the measurement area has a resolution of $W \times H$ and the projected fringe images are vertical. The wavelength of first fringe $\lambda_{1}$ is $W$. Since there is only one fringe covered the whole measurement area, no phase unwrapping is necessary, hence $\phi_{1}(x)=\Phi_{1}(x)$ and $m_{1}(x) \equiv 0$.

After the first fringe is projected, a series of fringe images with a wavelength decreased by a factor $N$ from its previous wavelength is used. In Zhang's research, the wavelength $\lambda_{k}=N \lambda_{k+1}, k=1,2,3, \ldots$ is used, where $N=1,2,3, \ldots$ As we know $\phi_{1}(x)=\Phi_{1}(x)$, since $\lambda_{1}=N \lambda_{2}$, it will have $\Phi_{2}=N \Phi_{1}$. Combining with Equation (11),

$$
m_{2}(x)=\text { Integer }\left[\frac{N \Phi_{1}(x)}{2 \pi}-\frac{\phi_{2}(x)}{2 \pi}\right]
$$

Here the operator Integer[] is to obtain the closest integer value. The wrapped phase, $\phi_{2}(x)$, can be unwrapped pixel by pixel by referring to the retrieved $m_{2}(x)$ :

$$
\Phi_{2}(x)=2 \pi m_{2}(x)+\phi_{2}(x)
$$

After $\Phi_{2}(x)$ is obtained, it can be used to correct $\phi_{3}(x)$. Hence in general, for $\lambda_{k}=\lambda_{k-1} / N$,

$$
m_{k}(x)=\text { Integer }\left[\frac{N \Phi_{k-1}(x)}{2 \pi}-\frac{\phi_{k}(x)}{2 \pi}\right]
$$

and

$$
\Phi_{k}(x)=2 \pi m_{k}(x)+\phi_{k}(x)
$$

Since the unwrapped phase is obtained pixel by pixel without accessing its neighbourhood pixels in the same phase map, and the noise of the longer wavelength will not significantly affect that of the shorter ones. Therefore, this technique can measure surface profile with arbitrary step height, and the measurement noise is close to that using a single-wavelength phase-shifting technique with the shortest wavelength.

\subsection{Multi-wavelength Spatial Shift Unwrapping Algorithm:}

In order to work out how to unwrap the spatial shift, a simple spatial shift unwrapping method has been proposed in [25], which unwraps the spatial shift using the neighbourhood pixels. However, this method has the limitation which cannot retrieve the correct shift distribution if the value difference of neighbourhood pixels is greater than $\lambda / 2$. Hence it cannot solve the unwrapping problem for those complex objects which have arbitrary step height.

To address this problem, we introduce a multiple wavelength unwrapping algorithm for spatial shift estimation approach based on Zhang's method. In this algorithm, we still assume the measurement area has a resolution of $W \times H$ and the projected fringe images are vertical. Then the wavelength of the fringe which projected in first time is selected to be $W$, that is $\lambda_{1}=W$. There is no need for spatial shift unwrapping since the single fringe stripe covers the whole measurement area. Hence we have $u_{1}(x)=U_{1}(x)$ and $m_{1}(x) \equiv 0$.

We then choose $\lambda_{2}=\lambda_{1} / N$. From equation (12), it is easy to find the real shift function $U(x)$ has no relationship with wavelength $\lambda$, that is, $U_{2}(x)=U_{1}(x)=U(x)$. Thus combining with Equation (13), we can have:

$$
m_{2}(x)=\text { Integer }\left[\frac{U_{1}(x)}{\lambda_{2}}-\frac{u_{2}(x)}{\lambda_{2}}\right]
$$

This means the wrapped spatial shift, $u_{2}(x)$, can be unwrapped by referring to the longer wavelength shift $U_{1}(x)$ pixel by pixel: 


$$
U_{2}(x)=\lambda_{2} m_{2}(x)+u_{2}(x)
$$

Because shift $U_{1}(x)$ is only used to obtain the integer $m_{2}(x)$, the noise of $U_{1}(x)$ does not significantly affect the noise of $U_{2}(x)$. After $U_{2}(x)$ is retrieved, we can use it to get $U_{3}(x)$, where $\lambda_{3}=\lambda_{2} / N$. So in general, for $\lambda_{k}=\lambda_{k-1} / N$, we have

$$
m_{k}(x)=\text { Integer }\left[\frac{U_{k-1}(x)}{\lambda_{k}}-\frac{u_{k}(x)}{\lambda_{k}}\right]
$$

and

$$
U_{k}(x)=\lambda_{k} m_{k}(x)+u_{k}(x)
$$

From Equation (20), we can find that the unwrapped spatial shift map is retrieved by referring to the longer wavelength shift map. Compare to those algorithms which use the neighbourhood pixels to do the unwrapping, this method can measure the surface profile with arbitrary step height. Since the noise of longer wavelength will not significantly affect the noise of shorter one, the measurement noise is close to the noise of using the shortest wavelength. This algorithm also has the advantage that a random noise reduction filter can be used to smooth the retrieved shift maps for longer wavelength hence increasing the robustness of the calculation.

\section{Experiments and Results}

In order to test the performance of the performance of the approach proposed in Section III, experiments were carried out in our laboratory. The experimental setup is shown in Figure 3. The digital projector used is HITACHI CP-X260, and camera is Duncan Tech MS3100. The digital camera is placed on top of the projector with a distance of $350 \mathrm{~mm}$. The distance between the camera lens and the reference plan is $1295 \mathrm{~mm}$. The resolution of the CCD camera is $1392 \times 1039$ pixels, and the field of vision for CCD camera is $250 \mathrm{~mm} \times 187 \mathrm{~mm}$. Hence, the equivalent spatial resolution is $0.1796 \mathrm{~mm} /$ pixel.

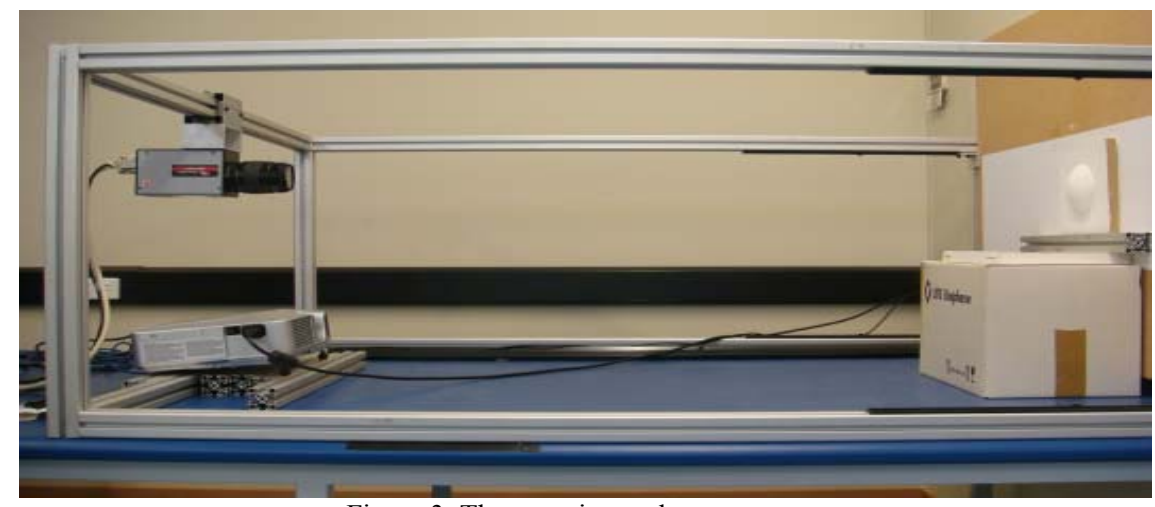

Figure 3. The experimental system setup

To verify the proposed method can measure the surface profile with arbitrary step height, we choose a mask as the measured object. A four-wavelength spatial-shifting algorithm with $\lambda_{1}=480$ and $N=2$ is used in our experiment. The unwrapped $u(x)$ is retrieved using Wu's method et al [23]. Figure 4 (a) shows the mask and Figures 4 (b) - (e) show the triangular fringe images of object with different wavelength. 


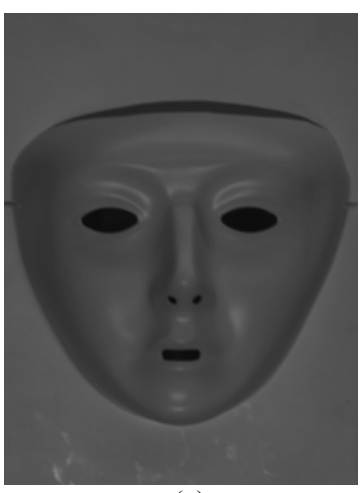

(a)

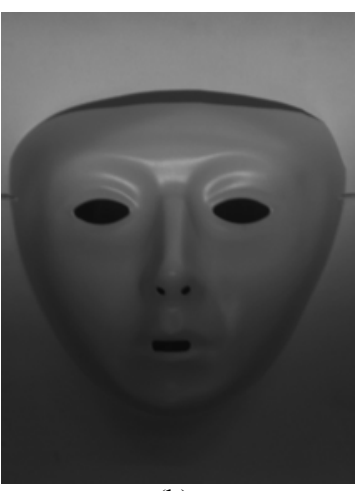

(b)

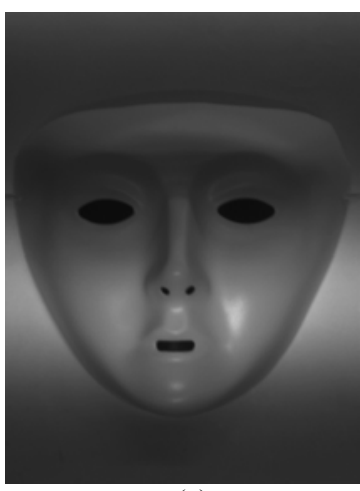

(c)

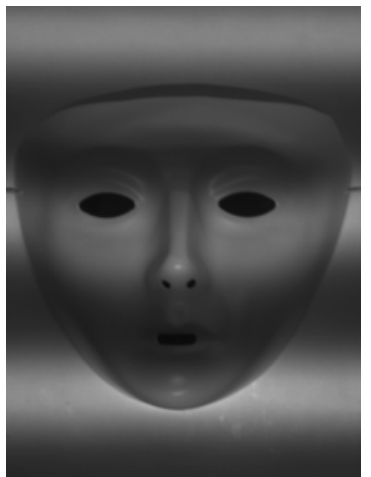

(d)

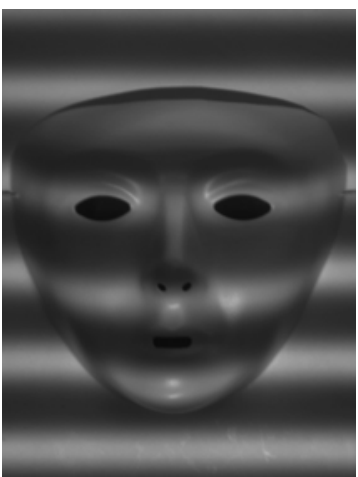

(e)

Figure 4. Captured triangular fringe images of objects with different wavelength. (a) object; (b) fringes image $(\lambda=480)$;

(c) fringes image $(\lambda=240)$; (d) fringes image $(\lambda=120)$; (e) fringes image $(\lambda=60)$;

Figure 5 shows the reconstructed 3D surface shape of the object using the proposed unwrapping method. It can be seen from these figures, the mask is reconstructed successfully. The height information for those places with high step drop (mask edge, nose part) is retrieved correctly. Some details, such as mouth and eyes part, are also recovered. This result demonstrated that the proposed method can successfully measure those complex objects with arbitrary step height.

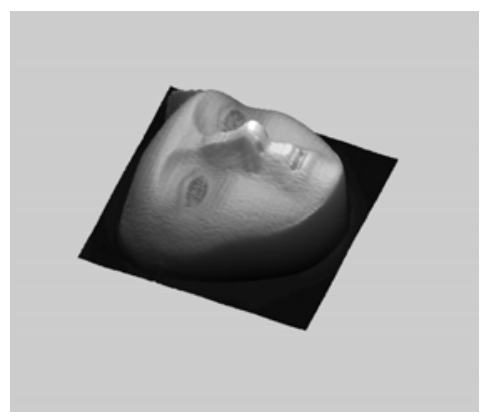

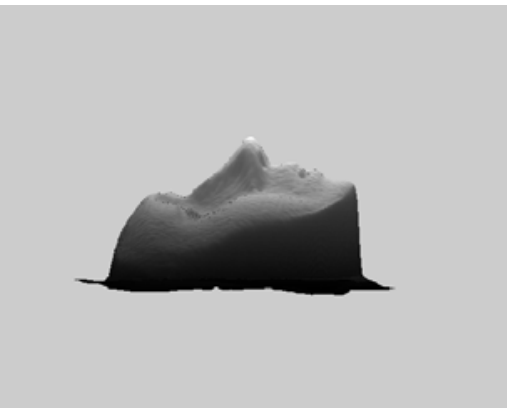

Figure 5. 3D reconstruct results

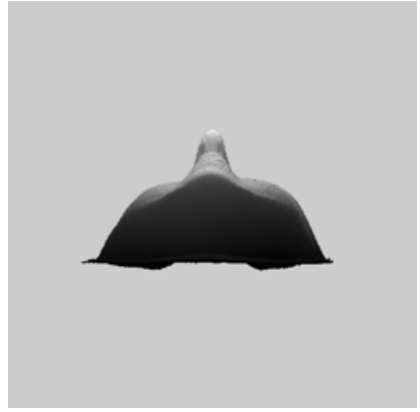




\section{Conclusion}

In this paper, we studied the spatial shift wrapping problem associated with SSE-based FPP. The problem arises as the result of fringe reuse (that is, fringes periodic light intensity variance), and the spatial shift can only be identified without ambiguity with the range of a fringe width. We presented a technique to carry out spatial shift unwrapping to remedy the problem. In order to test the performance, we also carried out experiments on an object with a mask which has complex surface shape. The results have shown the effectiveness of the proposed unwrapping technique.

\section{REFERENCES}

[1] X. Su and W. Chen, "Fourier transform profilometry: a review," Optics and Lasers in Engineering, vol. 35, pp. 263284, 2001.

[2] H. Zhang, M. J. Lalor, and D. R. Burton, "Spatiotemporal phase unwrapping for the measurement of discontinuous objects in dynamic fringe-projection phase-shifting profilometry," Applied Optics, vol. 38, pp. 3534-3541, June 1999.

[3] M. Halioua and H. C. Liu, "Optical three-dimensional sensing by phase measuring profilometry," Optics and Lasers in Engineering, vol. 11, pp.185-215, 1989.

[4] J. Li, H. Su, and X. Su, "Two-frequency grating used in phase-measuring profilometry," Applied Optics, vol. 36, pp. 277-280, Janurary 1997.

[5] X. Su, L. Su, W. Li, and L. Xiang, "New 3D profilometry based on modulation measurement," Proceedings of SPIE, Vol. 3853, pp. 1-7, 1998.

[6] S. Toyooka and M. Tominga, "Spatial fringe scanning for optical phase measurement," Optics Communications, Vol. 51, pp. 68-70, 1984.

[7] S. Toyooka and Y. Iwaasa, "Automatic profilometry of 3-D diffuse objects by spatial phase detection," Applied Optics, vol. 25, no. 10, pp. 1630-1633, May 1986.

[8] R. Rodriguez-Vera and M. Servin, "Phase locked loop profilometry," Optics and Lasers Technology, vol. 26, no. 6, pp. 393-398, 1994.

[9] D. M. Meadows, W. . Johnson, and J. B. Allen, "Generation of surface contours by moiré patterns," Applied Optics, vol. 9, no. 4, pp. 942-947, April 1970.

[10] A. Asundi and Z. Wensen, "Unified calibration technique and its applications in optical triangular profilometry," Applied Optics, vol. 38, no. 16, pp. 3556-3561, June 1999.

[11] C. Wust and D. W. Capson, "Surface profile measurement using color fringe projection," MVA, vol. 4, pp. 193-203, 1991.

[12] P. Huang, Q. Ho, F. Jin, and F. Chiang, "Colour-enhanced digitial fringe projection technique for high-speed 3-D surface contouring," Optics Engineering, vol. 38, pp. 1065-1071, 1999.

[13] A. J. Moore and F. Mendoza-Santoyo, "Phase demodulation in the space domain without a fringe carrier," Optics and Lasers in Engineering, vol. 23, pp. 319-330, 1995.

[14] J. Villa, M. Servin, and L. Castillo, "Profilometry for the measurement of 3-D object shapes based on regularized filters," Optics Communication, vol. 161, pp. 13-18, 1999.

[15] Y. Hu, J. Xi, E. Li, J. Chicharo, and Z. Yang, "Three-dimensional profilometry based on shift estimation of projected fringe patterns," Applied Optics, vol. 45, no. 4, pp. 678-687, February 2006.

[16] Y. Hu, J. Xi, J. Chicharo, W. Cheng, Z. Yang, "Inverse Function Analysis Method for Fringe Pattern Profilometry," IEEE Transactions on Instrumentation and Measurement, vol. 58, pp. 3305-3314, 2009.

[17] Y. Hu, J. Xi, J. Chicharo, and Z. Yang, "Optimal filtering based shift estimation for fringe pattern profilometry by generalized analysis model," in IEEE International Symposium on Intelligent Signal Processing and Communication Systems (ISPACS), Japan, December 2006.

[18] S. Zhang, "Phase unwrapping error reduction framework for a multiple-wavelength phase-shifting algorithm", Optics Engineering, vol. 48, pp. 1056011-1056018, 2009.

[19] M. Takeda, H. Ina, and S. Kobayashi, "Fourier-transform method of fringe-pattern analysis for computer-based topography and interferometry," Journal of the Optical Society of America A, vol. 72, pp. 156-160, 1982.

[20] M. Takeda and K. Mutoh, "Fourier transform profilometry for the automatic measurement of 3-D object shapes," Applied Optics, vol. 22, pp. 3977-3982, 1983.

[21] K. Itoh, “Analysis of the phase unwrapping algorithm”, Applied Optics, Vol. 21, Issue 14, pp. 2470-2470, July 1982. 
${ }^{[22]}$ D. C. Ghiglia, M. D. Pritt, Two-Dimensional Phase Unwrapping: Theory, Algorithms, and Software. John Wiley \& Sons, 1998.

[23] K. Wu, J. Xi, Y. Yu and Z. Yang, "3D profile measurement based on estimation of spatial shifts between intensity ratios from multiple-step triangular patterns", Optics and Lasers in Engineering, vol.51, pp.440-445, 2013.

[24] P. Jia, J. Kofman, and C. English, "Multiple-step triangular-pattern phase-shifting and the influence of number of steps and pitch on measurement accuracy," Applied Optics, Vol. 46, pp. 3253-3262 , 2007.

[25] P. Cao, J. Xi, J. Chicharo and Y. Yu, "A Fringe Period Unwrapping Technique for Digital Fringe Profilometry based on Spatial Shift Estimation", in Optical Inspection and Metrology for Non-Optics Industries, Proceedings of SPIE Vol. 7432 (SPIE, Bellingham, WA 2009) 743208. 